\title{
Las tierras negras en la mitología y arqueología de la vertiente sureste de la Sierra Nevada de Santa Marta, La Guajira
}

\author{
Dark Soils in the Mythology and Archaeology of the \\ Southeast of the Sierra Nevada de Santa Marta, La Guajira
}

\author{
José Vicente Rodríguez Cuenca* \\ Universidad Nacional de Colombia
}

\begin{abstract}
RESUMEN
Las tierras que actualmente habitan los indígenas de la Sierra Nevada de Santa Marta hacen parte de sus orígenes mitológicos y tradiciones culturales, en tanto fueron ocupadas milenariamente por sus ancestros, a quienes les proporcionaron un medio vital para su reproducción biológica y social. Su antigüedad solo se puede determinar mediante los estudios arqueológicos sistemáticos y los análisis fisicoquímicos de los suelos. El reporte de una tierra negra (antrosol) a orillas del río Ranchería en el sitio Santa Helena, en La Guajira, excavado durante el trabajo de arqueología preventiva en el marco del proyecto hidroeléctrico El Cercado (2008-2009), con dos fechas de radiocarbono, puede aportar nuevos datos a esta discusión.
\end{abstract}

Palabras clave: suelos antrópicos, patrón de ocupación, municipio de Distracción, La Guajira.

\begin{abstract}
$\overline{\text { ABSTRACT }}$
The land currently occupied by the Indians of the Sierra Nevada de Santa Marta is part of their mythological origins and cultural traditions, since it was occupied for millennia by their ancestors, to whom it provided a vital medium for biological and social reproduction. It's age can only be determined by systematic archaeological studies and physical-chemical soil analysis. The report of an Archaeological Black Earth (ABE) on the banks of the Rancheria River on the St. Helen a site in La Guajira, excavated during a preventive archeological study under the hydroelectric project El Cercado (2008-2009) with two radiocarbon dates, can bring new evidence to this discussion.
\end{abstract}

Keywords: archaeological black earth (ABE), settlement pattern, Distracción municipality, La Guajira.

Ph. D. en Antropología Física del Instituto de Antropología y Etnografía de Rusia. Profesor titular del Departamento de Antropología de la Universidad Nacional de Colombia. Recientemente publicó Rodríguez, José Vicente et al. 2016. Vida y muerte en el sur del Alto Magdalena, Huila. Bioarqueología y cambio social. Bogotá: Universidad Nacional de Colombia. jvrodriguezc@ unal.edu.co. 


\section{Introducción}

nvestigaciones etnográficas han señalado que los indígenas de la Sierra Nevada de Santa Marta (kággabas o koguis, ikas o arhuacos, wiwas o arsarios, kankuamos o atanqueros) conciben su territorio como un espacio vital que hay que preservar mediante rituales a las deidades tutelares, ofrendando "pagamentos" para asegurar la fertilidad de la sociedad en los sitios sagrados integrados por sepulcros de los mayores y otros lugares reverenciados (Uribe 1998, 81). Entre estos últimos tenemos las lagunas, algunos pozos grandes de ríos y quebradas, ciertas cuevas, rocas con petroglifos, centros ceremoniales y las extensas terrazas arqueológicas de cultivo, pues fue allí donde los "antiguos" les enseñaron a cultivar el maíz y otros productos, como lo demuestra la presencia de fragmentos de metates y manos de moler. En otros lugares de la sierra, como los valles de los ríos San Miguel y Ancho, también se han registrado montículos de piedra dispuestos para la fertilidad de las antiguas terrazas de cultivo, sitios considerados tabú y que, a pesar de contener suelos negros muy fértiles, no son aprovechados por los indígenas contemporáneos (Reichel-Dolmatoff 1985, II, 105-106).

En la mitología fundacional de los koguis de la Sierra Nevada de Santa Marta (SNSM), los indígenas le pidieron a Sintaná, el primer creador de la humanidad, que les proporcionara "tierra negra, la hija negra de la Madre, la buena tierra para la siembra”, pues las otras (arenosas, gredosas, amarillas) no eran tan benéficas para los cultivos (Preuss [1919] 1993, II: 10-11; Reichel-Dolmatoff 1985, I: 204-205). Sintaná accedió y con la ayuda de su compañero, el Viento, buscó por todos los rincones del mundo hasta que halló a una de sus hijas, aquella que representaba la "tierra negra de la Madre". De ella surgió la primera pareja de humanos en un templo construido por los cuatro señores (que representan los cuatro grupos indígenas de la Sierra Nevada: koguis, ijkas, wiwas, kankuamos), y de allí Sintaná salió a poblar el mundo kogui.

Con respecto a las plantas cultivadas, a la llegada de los conquistadores en el siglo XVI a las estribaciones noroccidentales de la SNSM, los cronistas anotaron que "de yuca y maíz es la cultura”, y que los indígenas preparaban cazabe con el almidón de yuca, y bollos y tortillas con el maíz; también tenían sementeras con cultivos de frutales, ahuyama y fríjol (Castellanos [1601] 1997, 497, 516-517, 535). En los fitolitos recuperados en excavaciones arqueológicas de Ciudad Perdida ${ }^{1}$ en

1 Área arqueológica caracterizada por un monumental conjunto de terrazas, muros, caminos y escaleras de piedra construidos por distintos pueblos prehispánicos, hoy convertida en el parque arqueológico Ciudad Perdida. 
la SNSM, se han registrado maíz, palmas y gramíneas no identificadas, fríjol, ají, batata, tabaco y algodón, que se cultivan actualmente, aunque no se ha detectado coca (la principal planta sagrada) (Giraldo 2010, 338).

La documentación arqueológica del uso de los suelos por parte de los indígenas prehispánicos de la SNSM incluye el análisis de fosfatos de las áreas libres del centro urbano Buritaca 200 (Ciudad Perdida), pues se ha establecido que en los antrosoles y lugares de depósito de basuras su concentración es más alta que en los sitios no habitados (Lleras 1987, 101-102). Sin embargo, la interpretación de los sitios rituales relacionados con la mitología de las comunidades indígenas del pasado requiere un análisis integral, que combine distintas estrategias de recolección de información en campo, desde la prospección intensiva para documentar el patrón de ocupación de un área determinada, hasta los cortes arqueológicos en área que aborden la estratigrafía paleoecológica, cultural y cronológica. La interpretación será más cercana a la realidad del momento analizado si se cuenta con personas sobrevivientes del lugar objeto de estudio que conserven la memoria de los pueblos que ocuparon antiguamente la zona, como nos sucedió durante un trabajo de arqueología preventiva en el área de influencia de un proyecto hidroeléctrico, en la vertiente sureste de la SNSM. Durante este trabajo, hallamos un suelo negro que por sus características fisicoquímicas podría corresponder a un antrosol y que, a juzgar por las estructuras de piedra que lo cubrían, fue sepultado y ritualizado para evitar su uso posterior.

Como parte de la estrategia etnográfica acudimos al acompañamiento permanente de un mamo (sabedor, sacerdote) wiwa ${ }^{2}$, nativo y conocedor de la región de Caracolí (Sabana Manuela) y de los sitios rituales que vinculan la Línea Negra, y con quien realizamos pagamentos alrededor de esta con el fin de pedir la autorización para ingresar a los sitios arqueológicos investigados y que iban a ser cubiertos por el agua de un embalse, antes, durante y después de las excavaciones. La Línea Negra es el territorio sagrado ancestral que circunda la SNSM y conecta varios sitios rituales donde los mamos realizan pagamentos según el significado de cada sitio: desde Valledupar, Cuestecita, Riohacha, río Ancho, Taganga, Fundación y Bosconia hasta llegar de nuevo a Valledupar.

Este artículo pretende aportar información a la discusión sobre el papel de las tierras negras para los indígenas prehispánicos de la vertiente sureste de la SNSM, región de contacto con las tradiciones culturales de la península de La Guajira, desde la perspectiva arqueológica apoyada en la interpretación etnográfica. La investigación se llevó a cabo en los años 2009-2010 en el área de influencia 
del proyecto hidroeléctrico El Cercado, ubicado entre Caracolí (llamado también Sabana Manuela) y Chorreras (municipio de Distracción, departamento de La Guajira). Durante el trabajo de campo tuvimos la oportunidad de recuperar información arqueológica de tres sitios con evidencias de una gran actividad ritual (El Cercado, El Espinito y Santa Helena) y documentar la existencia de una tierra negra (antrosol) a orillas del río Ranchería en el sitio Santa Helena, en La Guajira. En el 2009, los habitantes de Caracolí afirmaron que el lugar de este estudio fue abandonado hacia mediados del siglo XX por indígenas de origen wiwa.

\section{Las tierras negras en la arqueología}

Las terras pretas, tierras negras, terras pretas do indio o tierra negra de indio (TPI) son suelos antropogénicos muy antiguos, producto de una intensa actividad para la agricultura de subsistencia mediante el sistema de policultivos. También han sido denominadas archaeological black earth (ABE), Amazon dark earths (ADE) o Indian black earth (IBE), y se destacan por ser más productivas que los suelos circundantes como consecuencia de su enriquecimiento con microflora (Pinheiro et al. 2006, 282). Los antrosoles provienen de la acumulación de desechos domésticos (estiércol, cerámica, restos de carbón, material lítico, ceniza de alimentos) que incorporan sustancias orgánicas que les permiten sostener una mayor actividad biológica y una alta capacidad de retención de nutrientes. Estos suelos son indicadores de un área óptima para los asentamientos indígenas. Tienen horizontes A gruesos, cuyo color varía entre negro (10YR 2/1) en la escala de Munsell y ceniza muy oscuro, y poseen elevados tenores de fósforo (P), calcio (Ca), magnesio $\left(\mathrm{Mg}\right.$ ), potasio $(\mathrm{K}), \mathrm{pH}$, micronutrientes y flujo de $\mathrm{CO}_{2}$ (Andrade 1990, 78-79; Botero 2001, 42; Morcote, Mora y Franky 2006, 83-90; Morcote y León 2012, 62; Pinheiro et al. 2006, 286).

En el ámbito arqueológico en Colombia, estos suelos se han registrado en la Amazonía y en la Orinoquía, en terrazas antiguas y piedemonte de ríos de agua clara cerca de rápidos, como el Caquetá, Guaviare-Guayabero y el interfluvio Putumayo-Amazonas (Andrade 1990; Botero 2001; Mora et al. 1990; Morcote 2008), en el Igarapé Takana, Leticia (Morcote y León 2012), y en otras partes de la Amazonía brasileña (Geraldes et al. 2006; Morán 1990; Pinheiro et al. 2006; Rebellato y Grosch 2006). También son sugerentes los elevados contenidos de fósforo total (hasta 4.810 p. p. m.), de calcio (hasta 23 gr) y de materiales arqueológicos contenidos en suelos negros del Parque Arqueológico de Facatativá, 
Cundinamarca (Rodríguez 2015, 65). En el Valle del Dorado de Calima-Darién, en el Valle del Cauca, también se reportan suelos negros antropogénicos con características fisicoquímicas de antrosoles, como parte de la construcción social del paisaje prehispánico (Salas 2011). Es probable que, en los antiguos huertos de Ciudad Perdida y Pueblito en la Sierra Nevada de Santa Marta, los indígenas construyeran suelos negros en las áreas libres aledañas a los centros urbanos, a juzgar por las evidencias arqueológicas, paleobotánicas y por el análisis de fosfatos (Giraldo 2010, 270; Lleras 1987, 114).

Investigaciones etnográficas en el Amazonas han demostrado que las tierras negras son parte de territorios administrados por chamanes, con un alto contenido ritual. Allí la historia se inscribe en el paisaje mediante referencias topográficas en los mitos de creación y en la tradición oral que se asocia con los antiguos lugares sagrados (Hugh-Jones 2012, 38; Van der Hammen 1992, 110; Vickers 1989, 82). En estos espacios las relaciones entre los humanos y los seres de la naturaleza son parte del tejido social, por lo que algunas plantas y animales se conciben como parientes cercanos, comadres y vecinos (Hugh-Jones 2013; Reichel-Dolmatoff 1997; Van der Hammen 1992). Para los pueblos indígenas que habitan la SNSM, la base de la estructura ritual es la fertilidad, mediante la cual se recibe (alimentos, hijos, salud, bienestar) y se ofrece (por medio de ofrenda o sacrificio) alimento (energía) para mantener la vitalidad en el mundo. La ofrenda más importante es a la madre universal, productora de toda la vida, la personificación de la fertilidad y de la naturaleza misma (Uribe 1998, 62) 3 .

En Colombia, la mayoría de registros de antrosoles se han producido en la Amazonía y en la Orinoquía. Su presencia en una terraza coluvio-aluvial del altomedio río Ranchería en La Guajira, vertiente sureste de la SNSM, con evidencias de ritualización mediante estructuras de piedras alineadas (según nos informó el mamo que nos acompañó), constituye el primer reporte arqueológico para esta región. Este fue registrado con la metodología del reconocimiento sistemático intensivo (Drennan 2000, 98) y complementado mediante cortes estratificados en área y análisis fisicoquímico de suelos, cuyos resultados constituyen la principal contribución a la arqueología regional. El objetivo de este artículo es documentar la existencia de un antrosol, su relación con el patrón de ocupación de la terraza de Santa Helena y su abandono de manera ritual para impedir su uso posterior, a la luz de nuevas fechas de radiocarbono (véanse figura 1, tabla 2), en una zona que en su momento constituyó una de las principales despensas agrícolas de San Juan del Cesar y que hoy está cubierta por las aguas de la represa El Cercado. 


\section{Arqueología de La Guajira}

A juzgar por la tipología cerámica recuperada, el sitio objeto de esta investigación arqueológica tiene una fuerte relación con las tradiciones culturales de la península de La Guajira, por lo que se mencionarán los tres grandes periodos de su desarrollo cultural prehispánico: Precerámico (VIII-II milenio a. C.), fase Hornoide (I milenio a. C. a siglo V d. C.) y fase Ranchoide (siglos VI-XVI d. C.). Se han registrado evidencias de estas tres fases de ocupación en la parte media de la llanura aluvial del río Ranchería, entre las poblaciones de Distracción y Albania (Ardila 1996; Langebaek, Cuéllar y Dever 1998; Reichel-Dolmatoff 1986). Sin embargo, la zona de mayor densidad está representada en las terrazas coluvioaluviales de la parte baja del alto río Ranchería en el sitio Santa Helena (municipios de Caracolí y Distracción), antiguo territorio de grupos afines a los del Valle de Upar, como los itotos (Castellanos [1601] 1997; De Aníbal et al. [1578] 1983), y de los wiwas históricos, cuyo principal centro era Marokaso (Setkuá), al noroeste de Caracolí (Sabana Manuela) (Isaacs [1883] 1951; Reclus 1992; Vinalesa 1952). Estos asentamientos se basaron en la alta fertilidad de sus suelos, lo que posibilitó el desarrollo de la agricultura, la elaboración de antrosoles y la existencia de ocupaciones humanas relativamente densas. Mientras que para la cuenca media se ha propuesto que hacia el siglo XIII d. C. se habría presentado una desocupación humana relacionada con un ambiente más seco (Langebaek, Cuéllar y Dever 1998, 49; Reichel-Dolmatoff 1986, 197), en las terrazas de ambas márgenes del río Ranchería, en el municipio de Distracción, se observa una ocupación ininterrumpida hasta mediados del siglo XX, cuando indígenas sobrevivientes de la debacle del siglo XVI originada por la conquista española enterraron de manera ritual un antrosol mediante hiladas de piedra dispuestas en la superficie, acompañadas de entierros humanos (véase figura 4) (Rodríguez, Cifuentes y Aldana 2010). Asimismo, en la vertiente noroeste de la Sierra Nevada de Santa Marta, según las recientes investigaciones arqueológicas, se aprecia una ocupación ininterrumpida entre los periodos Neguanje (400-1100/1200 d. C.) y Tairona (1100/1200 a 1600 d. C.) (Dever 2007; Giraldo 2010; Langebaek 2005).

\section{La terraza de Santa Helena}

A raíz de la construcción de la represa El Cercado sobre el río Ranchería bajo la supervisión del Instituto Colombiano de Desarrollo Rural (Incoder), se puso 


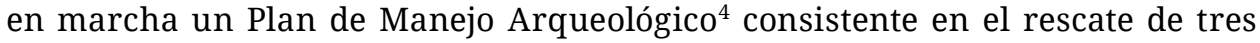
sitios: El Cercado, El Espinito y Santa Helena (figura 1). Los sitios se ubican en un valle entre el piedemonte de la SNSM y el río Ranchería, entre los municipios de Distracción y Caracolí (zona de Sabana Manuela), a 400 m s. n. m. Este valle aluvial presenta, a su vez, tres grandes paisajes, que incluyen la llanura aluvial con las vegas del río en la parte más baja, terrazas aluviales con tres niveles y el piedemonte de la sierra. La quebrada El Pital, que antes surcaba por el centro de la terraza para desembocar casi perpendicular al río Ranchería, cambió hace muchos años su cauce hacia el borde suroeste, lo que generó un abanico fluvial rico en sedimentos arrastrados desde la sierra que cubrieron toda la terraza con suelos fértiles. El antiguo cauce tenía agua permanente y dejaba depósitos de materiales gruesos durante el invierno y finos en el verano. Dado que esta agua es menos correntosa que la del río y más limpia, los pobladores prehispánicos prefirieron esta fuente a la del río Ranchería. Por su lado, la población prehispánica de la parte baja acudía más a los recursos del río. Con el tiempo, debido a la acumulación de materiales producidos por el abanico fluvial, el agua corría con menos intensidad, surcando de vez en cuando por la terraza. Estas condiciones fueron aprovechadas, especialmente para la agricultura, por los grupos de la fase Hornoide que habitaron esta región, desde inicios del I milenio d. C. (tabla 1).

Tabla 1. Dataciones radiocarbónicas obtenidas en la llanura aluvial del río Ranchería

\begin{tabular}{|c|c|c|c|c|c|}
\hline Beta & Fecha & Calibrada & Sitio & Periodo & Autor \\
\hline 7.281 & $413 \pm 50$ a. C. & 753 a 695 a. C. & $\begin{array}{l}\text { Río IV El } \\
\text { Palmar }\end{array}$ & Loma & Ardila (1996) \\
\hline 8.554 & $401 \pm 60$ a. C. & 481 a 448 a. C. & Patilla III & Loma & Ardila (1996) \\
\hline 7.280 & $90 \pm 200$ a. C. & $\begin{array}{l}290 \text { a. C. a } \\
110 \text { d. C. }\end{array}$ & $\begin{array}{l}\text { Río III El } \\
\text { Palmar }\end{array}$ & Loma & Ardila (1996) \\
\hline 145.849 & $0 \pm 70$ d. C. & $\begin{array}{l}30 \text { a. C. a } \\
120 \text { d. C. }\end{array}$ & Portacelli & Horno & Socarrás (2003) \\
\hline 278.824 & $160 \pm 40 \mathrm{~d} . \mathrm{C}$ & $70-250$ d. C. & $\begin{array}{l}\text { S. Helena UE5 } \\
60-70 \mathrm{~cm}\end{array}$ & Horno & Presente reporte \\
\hline 145.848 & $380 \pm 70$ d. C. & 410 a 570 d. C. & Portacelli & Horno & Socarrás (2003) \\
\hline 4.843 & $585 \pm 75$ d. C. & 510 a 650 & Patilla II & Portacelli & Ardila (1996) \\
\hline
\end{tabular}

$4 \quad$ Según lo exigido por la Ley General de Cultura (Ley 397 de 1997). 


\begin{tabular}{|c|c|c|c|c|c|}
\hline Beta & Fecha & Calibrada & Sitio & Periodo & Autor \\
\hline 7.284 & $930 \pm 110$ d. C. & 820 a 1040 & $\begin{array}{l}\text { Río IV El } \\
\text { Palmar }\end{array}$ & Portacelli & Ardila (1996) \\
\hline 146.378 & $970 \pm 70$ d. C. & 1000 a 1160 & Oreganal & Portacelli & Socarrás (2003) \\
\hline 7.283 & $\begin{array}{l}1046 \pm 190 \\
\text { d. } C .\end{array}$ & 895 a 915 & $\begin{array}{l}\text { Río IV El } \\
\text { Palmar }\end{array}$ & Portacelli & Ardila (1996) \\
\hline 259.732 & $1180 \pm 60$ d. C. & $1160-1300$ & El Cercado UE7 & Portacelli & $\begin{array}{l}\text { Rodríguez, Cifuen- } \\
\text { tes y Aldana (2010) }\end{array}$ \\
\hline 259.740 & $1200 \pm 70$ d. C. & $1160-1320$ & El Cercado UE7 & Portacelli & Presente reporte \\
\hline 7.282 & $1225 \pm 50$ d. $C$ & 1174 a 1276 & $\begin{array}{l}\text { Río IV El } \\
\text { Palmar }\end{array}$ & Portacelli & Ardila (1996) \\
\hline 8.552 & $1298 \pm 80$ d. C. & 1279 a 1328 & $\begin{array}{c}\text { Paredón I, } \\
\text { Oreganal }\end{array}$ & Portacelli & Ardila (1996) \\
\hline 8.553 & $1302 \pm 70$ d. C. & 1286 a 1398 & $\begin{array}{l}\text { Paredón I } \\
\text { Oreganal }\end{array}$ & Portacelli & Ardila (1996) \\
\hline 259.733 & $1310 \pm 60$ d. C. & $1270-1420$ & El Cercado UE7 & Portacelli & $\begin{array}{l}\text { Rodríguez, Cifuen- } \\
\text { tes y Aldana ( } 2010)\end{array}$ \\
\hline 259.734 & $1350 \pm 60$ d. C. & $1280-1430$ & El Espinito 3 C5 & $\begin{array}{l}\text { Portacelli } \\
\text { y Tairona }\end{array}$ & $\begin{array}{l}\text { Rodríguez, Cifuen- } \\
\text { tes y Aldana (2010) }\end{array}$ \\
\hline 144.494 & $1430 \pm 40$ d. C. & 1405 a 1430 & Oreganal & Portacelli & Socarrás (2003) \\
\hline 259.731 & $1460 \pm 60 \mathrm{~d} . \mathrm{C}$ & $1390-1480$ & El Cercado UE7 & Portacelli & $\begin{array}{l}\text { Rodríguez, Cifuen- } \\
\text { tes y Aldana (2010) }\end{array}$ \\
\hline 278.826 & $1700 \pm 40$ d. C. & $\begin{array}{c}1520-1590,1620- \\
1670,1770-1800 \\
1940-1950 \text { d. C. }\end{array}$ & $\begin{array}{l}\text { S. Helena } \\
\text { UE8/9 60-80 } \\
\mathrm{cm}\end{array}$ & $\begin{array}{c}\text { Portacelli y } \\
\text { Contemporáneo }\end{array}$ & Presente reporte \\
\hline 259.735 & $1950+$ & $1950+$ & $\begin{array}{c}\text { El Espinito } 2 \\
\text { UE1 }\end{array}$ & Contemporáneo & $\begin{array}{l}\text { Rodríguez, Cifuen- } \\
\text { tes y Aldana (2010) }\end{array}$ \\
\hline 259.736 & $1950+$ & $1950+$ & El Espinito $3 \mathrm{C1}$ & Contemporáneo & $\begin{array}{l}\text { Rodríguez, Cifuen- } \\
\text { tes y Aldana ( } 2010 \text { ) }\end{array}$ \\
\hline
\end{tabular}

Fuente: elaboración propia. 
Figura 1. Ubicación de los sitios arqueológicos cubiertos por la represa El Cercado (área subrayada) (Rodríguez, Cifuentes y Aldana 2010)

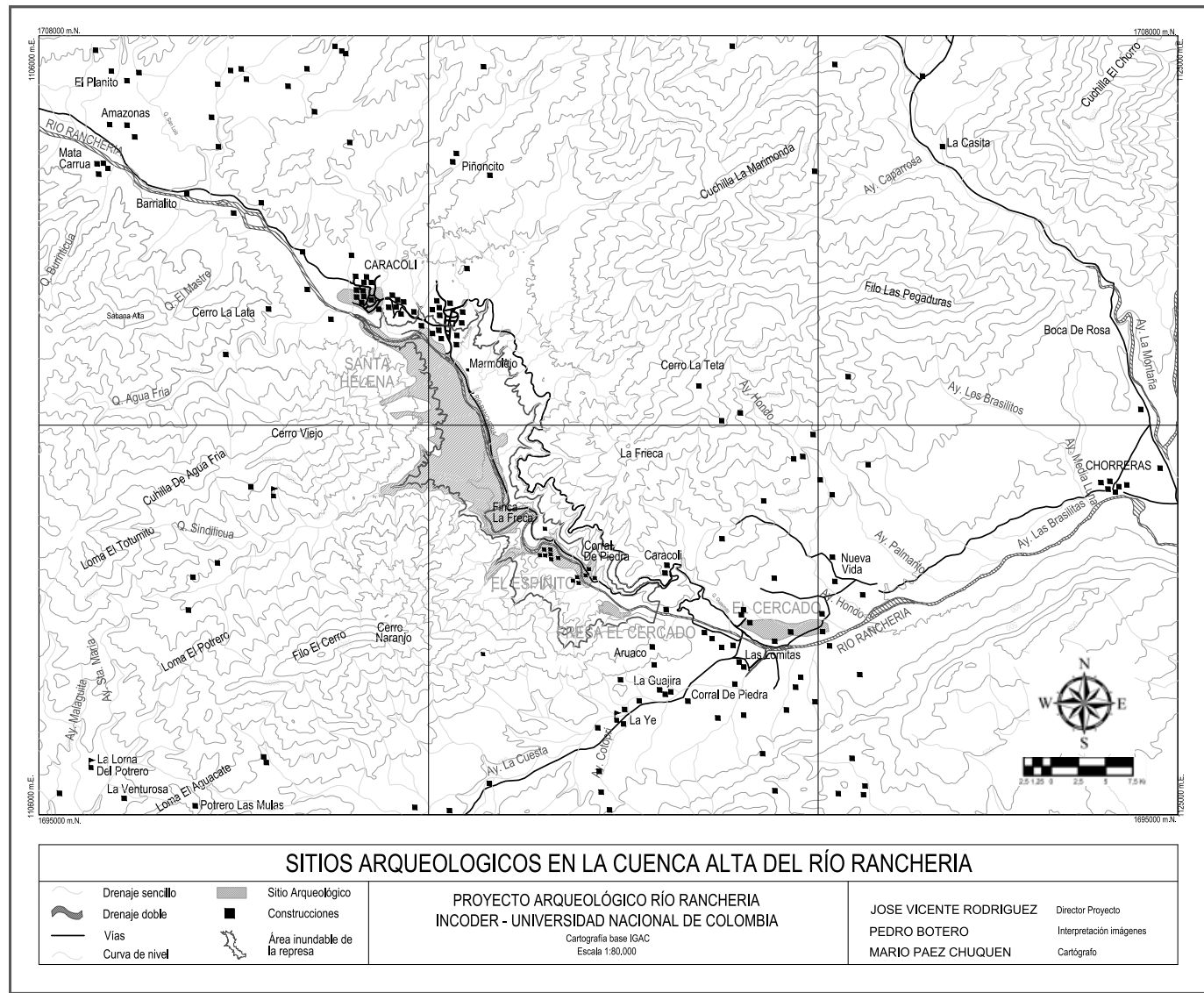

Fuente: Rodríguez, Cifuentes y Aldana (2010).

\section{La prospección sistemática}

En Santa Helena se hizo un reconocimiento sistemático intensivo (Drennan 2000, 98) con el fin de documentar el patrón de ocupación de la terraza, mediante pozos de sondeo de $100 \times 100 \times 180 \mathrm{~cm}$ distanciados cada $50 \mathrm{~m}$, para un total de 92 sondeos distribuidos en 19 líneas, que cubrieron la totalidad de la terraza aluvial de las fincas Santa Helena, El Porvenir, El Melao y Palonegro (que fueron adquiridas por el Incoder a sus anteriores propietarios y actualmente están cubiertas por la represa El Cercado), y que registraron todos los momentos de ocupación en el ámbito cronológico (figura 1). 
La distribución de la cerámica de todos los sondeos se definió por niveles arbitrarios cada $20 \mathrm{~cm}$, teniendo en cuenta la estratigrafía de los suelos, en los que, por lo general, en los primeros $40 \mathrm{~cm}$ del perfil se registra la ocupación tardía (Ranchoide), y en el nivel 40-100 cm la ocupación temprana (Hornoide). La primera ocupación inició aproximadamente hacia los 70-80 cm de profundidad, con un suelo de color 10YR 4/3 (pardo oscuro), hasta los 40-60 cm, en donde se acumula el 39,1\% del material cerámico recolectado de los sondeos; edafológicamente se ubica en un horizonte Abp3 de color parduzco oscuro (10YR 3/3), perturbado por la presencia del material cultural (cerámica y líticos). Entre 0-40 cm encontramos evidencias de una ocupación más densa (60,9\% de los materiales) y se refleja un uso intensivo del suelo (Abp2) (tabla 2).

Tabla 2. Distribución de la cerámica de los sondeos en Santa Helena por niveles y ocupación

\begin{tabular}{c|c|c|c|c} 
Nivel (cm) & Cerámica & $\begin{array}{c}\text { Porcentaje } \\
(\%)\end{array}$ & $\begin{array}{c}\text { Ocupación } \\
\text { predominante }\end{array}$ & $\begin{array}{c}\text { Incremento del } \\
\text { porcentaje (\%) }\end{array}$ \\
\hline $0-40$ & 1.960 & 60,9 & Ranchoide & 55,9 \\
\hline $40-100$ & 1.257 & 39,1 & Hornoide & \\
\hline Total & 3.217 & 100 & & \\
\hline
\end{tabular}

Fuente: elaboración propia.

Al parecer, la ocupación de la terraza fue multifuncional y la población prehispánica se concentró a lado y lado del antiguo camino que existía entre El Pital en la SNSM y el río Ranchería. Las líneas de sondeo 1, 2 (margen norte) y 15 y 16 (margen sur), en el centro de esta unidad de paisaje (posee los suelos más húmedos, sueltos, arenosos y fértiles), reúnen casi la totalidad de la muestra cerámica recolectada (91,9\%), con un total de 2.957 fragmentos cerámicos en 26 sondeos, lo que promedia 113,7 fragmentos por $\mathrm{m}^{3}$, o 739,3 fragmentos por hectárea. Cronológicamente corresponde a un asentamiento que existió desde inicios del I milenio d. C. y que hasta el año 2009 (tabla 1), cuando se construyó la represa El Cercado, constituyó la más importante despensa agrícola para Distracción y San Juan del Cesar (La Guajira), debido a su alta productividad.

\section{Excavaciones en área}

Para complementar la información recabada durante la prospección sistemática se realizaron nueve cortes estratificados (unidades de excavación [UE]), con el fin de dar cuenta de la distribución temporal de los materiales. De cada horizonte de 
suelos se tomaron muestras para el respectivo análisis fisicoquímico, aunque solo se referencian los tres cortes $(5,8$ y 9) más productivos en cuanto a fechados y material cultural (tabla 3).

\section{Unidad de excavación 5}

Se localiza en la parte central de la terraza (figuras 1 y 2). El corte se excavó de 200 x 200 x $140 \mathrm{~cm}$ mediante niveles convencionales de $10 \mathrm{~cm}$, y se sometió a cernido todo el suelo con el fin de recuperar evidencias culturales y ecofactos. Los análisis fisicoquímicos de los suelos indican que fueron objeto de uso ininterrumpido para el cultivo intensivo debido a las excelentes condiciones agrícolas, tanto por los atributos fisicoquímicos, como por la cercanía a una fuente de agua que podía irrigar los cultivos y sustentar a la gente que dependía de los productos de esta tierra.

Figura 2. UE 5 de Santa Helena en el nivel $70 \mathrm{~cm}$

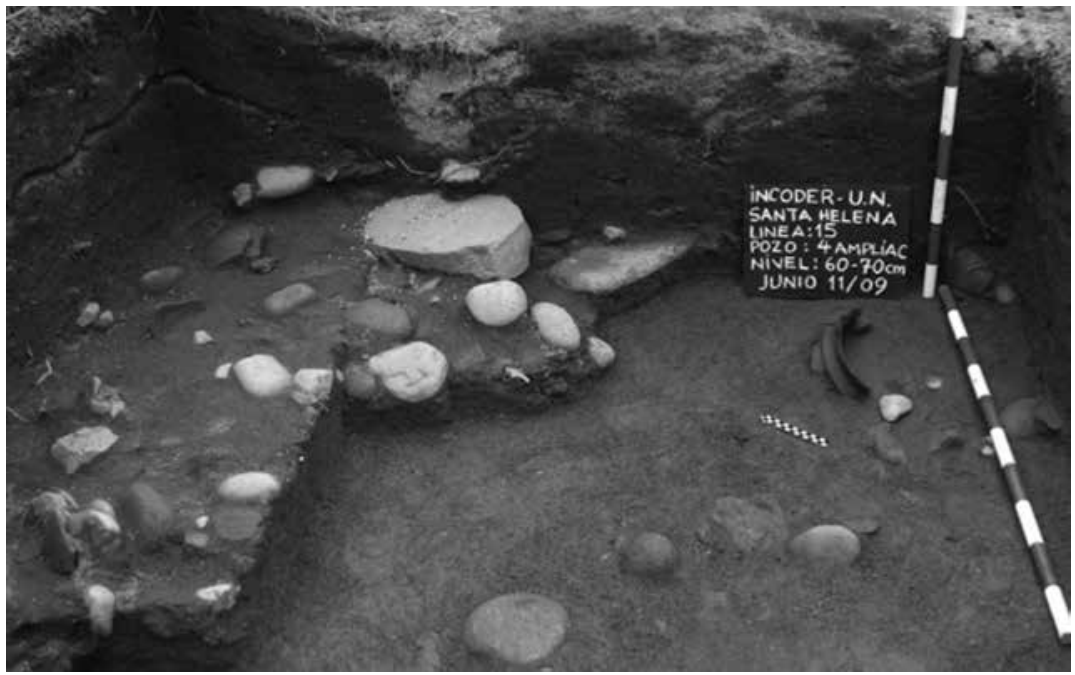

Fuente: elaboración propia.

El horizonte 40-70 $\mathrm{cm}$ posee la mayor densidad de material cerámico (72,8\%), correspondiente a la tradición Hornoide (figura 3), de donde se obtuvo una datación mediante radiocarbono (beta 278.824) de $160 \pm 40$ d. C. (a 60-70 cm de profundidad) que corresponde al momento de la mayor presencia humana. Esta es la fecha más antigua registrada para el piedemonte de la SNSM (tabla 1) (véanse fechados recientes para la SNSM en Dever 2007 y Giraldo 2010). La distribución cerámica presenta varios tipos donde predominan el café liso (36,2\%), naranja liso 


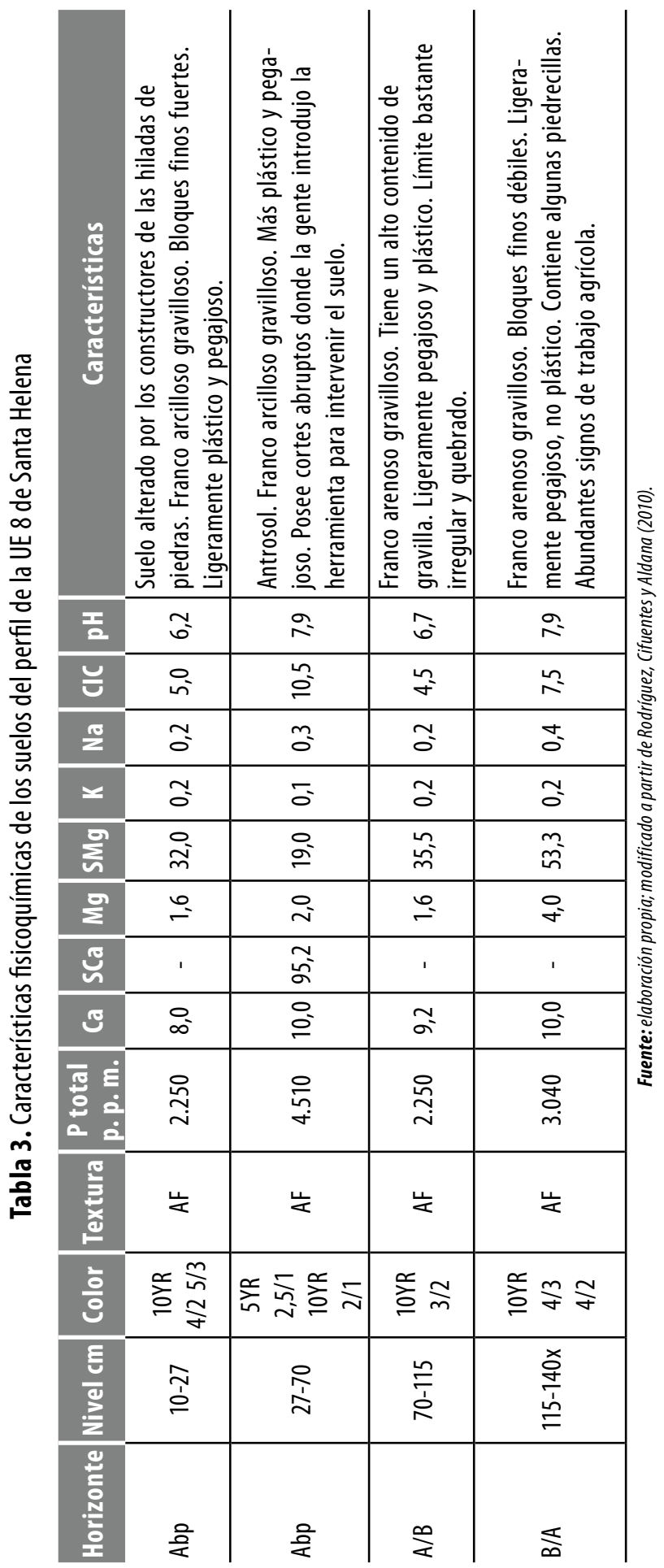


$(21,1 \%)$, crema a naranja $(17,8 \%)$ y rojo grano medio $(8,3 \%)$, además de café pulido, esgrafiado y rojo pulido, cuyas frecuencias son mayores en el nivel inferior. Es decir, la primera ocupación se caracteriza por esos tipos cerámicos, por una mayor densidad de población y una mayor amplitud cronológica, que se pudo iniciar hacia finales del I milenio a. C. La última ocupación $(0-40 \mathrm{~cm})$ se caracteriza por los tipos Portacelli y Tairona negro pulido y una baja densidad de materiales (solamente $0,2 \%$ ).

Figura 3. Estructura de piedra que recubre la tierra negra, con ofrendas compuestas de vasijas y manos de moler de la terraza 1 de Santa Helena

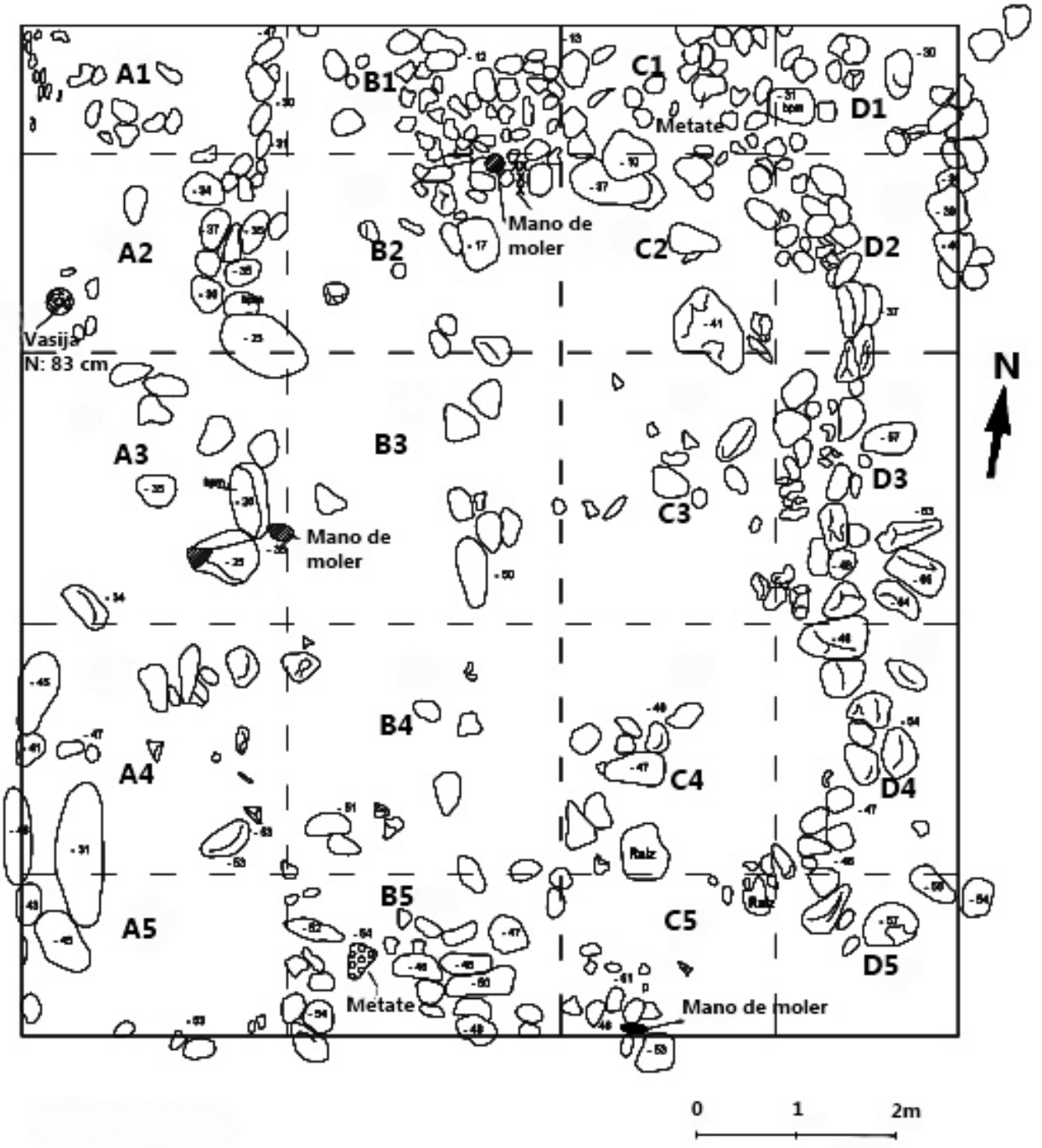

Fuente: Rodríguez, Cifuentes y Aldana (2010). 
Con respecto a la industria lítica, se pueden diferenciar los artefactos pulidos (hachas, manos, yunques, cinceles) y de molienda (cantos con bordes desgastados) que alcanzan un 21,3\%; los elementos lascados (lascas, microlascas, cortadores, raspadores, perforadores) conforman el 78,7\% del total de la muestra. Al igual que para la distribución cerámica, los líticos predominan en el nivel 40-100 cm con un 77,4\% del total registrado, mientras que en el nivel 0-40 cm se presenta solo un $22,6 \%$.

A juzgar por las características fisicoquímicas del suelo, en el horizonte Abp3 (con tenores medios de fósforo total -894 p. p. m. - estructura degradada por compactación), ubicado estratigráficamente entre 40-60 cm y cronológicamente hacia el siglo II d. C., se presenta un uso agrícola intensivo, lo que se confirma por los tipos de artefactos (molienda y pulidos) y cerámica para servir (cuencos) y cocinar (ollas globulares y subglobulares), como se ha demostrado en el sector noroeste de la SNSM, a partir de análisis de polen y fitolitos (Giraldo 2010, 270).

De esta manera, la UE 5 de Santa Helena tiene las evidencias más antiguas de las ocupaciones humanas del borde sureste de la SNSM, con estilos cerámicos propios del Bajo Magdalena y Ranchería Medio y, luego, de la tradición tairona.

\section{Unidad de excavación 8}

En el extremo noroeste y más elevado del área central de Santa Helena (figura 3) se localizó un sitio con evidencias que se remontan desde la tradición Hornoide hasta mediados del siglo XX, con predominancia de la tradición Ranchoide. En un área de más de $50 \mathrm{~m}^{2}$ se observan estructuras de piedra alineadas que cubrían un antrosol (terrapreta) (figura 3), horizonte que se ubica entre 30-70 cm de profundidad, con límites muy ondulados por la intervención humana y tenores elevados de fósforo total (4.510 p. p. m.), calcio (10 gr), magnesio (2 gr), potasio (0,14 gr) y micronutrientes. El contenido de calcio es más del doble, lo mismo que el de sodio y el de carbón orgánico, comparados con los otros suelos de la terraza de Santa Helena. Las cantidades de fósforo total son muy altas (más de 4.510 p. p. m.), no solo en comparación con los suelos de esta terraza, sino con los de El Espinito y otros suelos antropogénicos de la Amazonía (Geraldes et al. 2006, 299; Morcote 2008, 84; Morcote y León 2012, 65), aunque cercanos a los reportados por Lleras $(1988,109)$ en Buritaca 200 (Ciudad Perdida, vertiente noroeste de la SNSM).

En este sector de la terraza, donde precisamente se registró el suelo negro, se colocaron cantos rodados de tamaño uniforme transportados desde el río Ranchería, dispuestos de manera organizada, acompañados de vasijas (urnas funerarias), manos de moler y metates, para indicar una actividad intencional, de tipo ritual, según las tradiciones de la SNSM (figuras 3 y 4). 
Figura 4. UE 9 de la terraza 1 de Santa Helena con piedras dispuestas intencionalmente y vasijas de ofrenda (Rodríguez, Cifuentes y Aldana 2010)

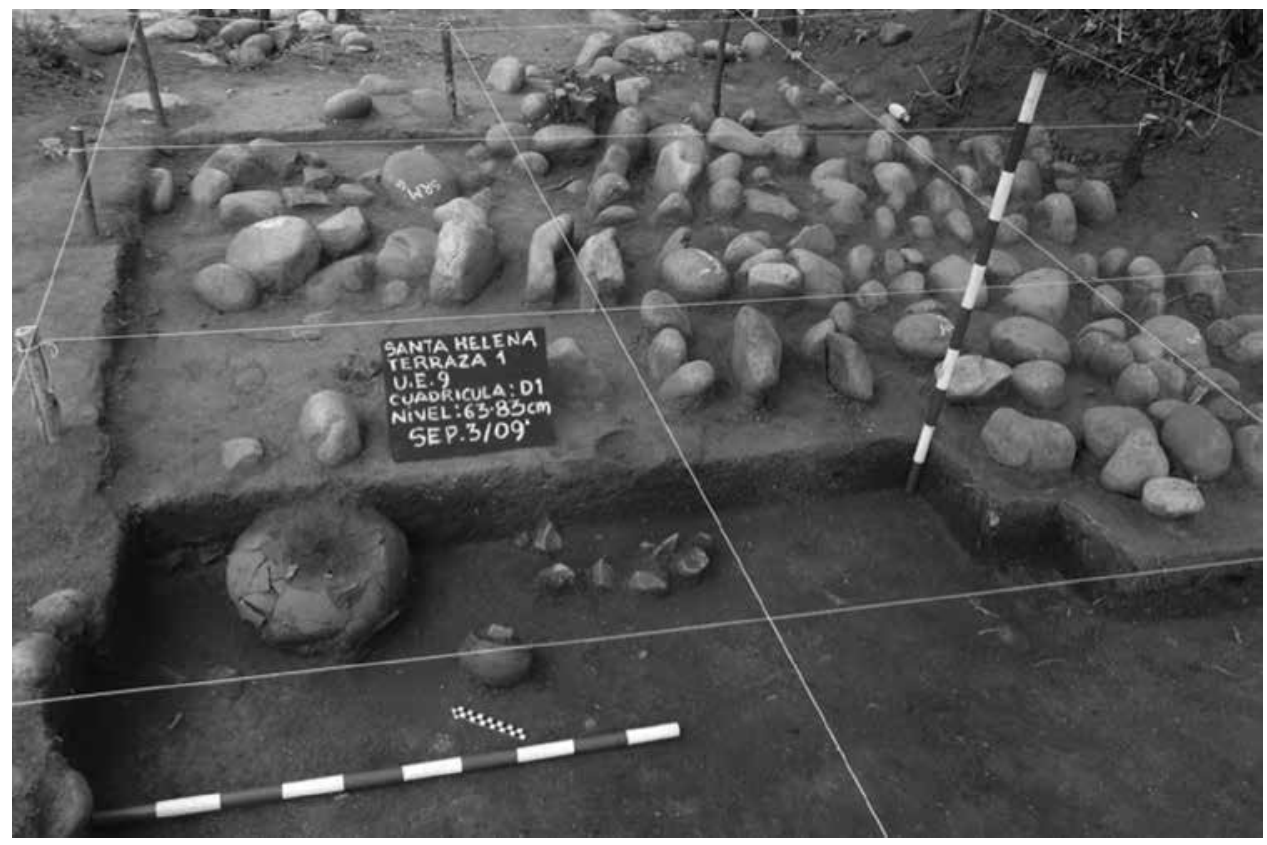

Fuente: Rodríguez, Cifuentes y Aldana (2010).

Llama la atención que los tipos cerámicos predominantes debajo del antrosol son de la tradición Hornoide (naranja liso, $40 \%$; café liso, 22,7\%; café pulido, $12 \%$; crema a naranja, 10,7\%), mientras que las fechas calibradas (beta 278.826) de una muestra de carbón obtenida entre $60-80 \mathrm{~cm}$ de profundidad señalan distintos momentos de intervención del suelo: 1520-1590, 1620-1670, 1770-1800, 19401950 d. C. (tabla 1). Como los antrosoles se forman por la permanente intervención humana mediante la aportación de desechos domésticos, las fechas pueden estar indicando intervenciones humanas durante varios momentos históricos entre el siglo XVI y mediados del XX cuando, antes de abandonar el sitio por la presión de colonos guajiros, los indígenas que vivían allí sepultaron un suelo considerado sagrado según la tradición serrana. Incluso, entre las piedras se registraron objetos modernos como huesos de asno, balines y una cruz de madera engastada en plata.

\section{Unidad de excavación 9}

Este sitio se ubica en la parte más elevada de la terraza aluvial, donde se registró una alta concentración de artefactos líticos (272 en total), especialmente, 
instrumentos lascados (86\%) y en menor medida, pulidos (14\%). Entre 0-60 cm de profundidad predomina la ocupación Ranchoide, en particular, el tipo cerámico Portacelli (café burdo, naranja, crema) con el 36,9\%, seguido del negro Riohacha (14,5\%). En el nivel 60-180 cm prevalece la ocupación Hornoide con predominancia del tipo café liso (13,5\%). Las hachas tabulares y las bases para molienda se encuentran fragmentadas, evidencia de que el sitio se aprovechó para enterrar desechos de artefactos y para labores domésticas, a juzgar por la alta frecuencia de los instrumentos lascados. Los tenores de fósforo total no son tan elevados como los del corte 8 (oscilan entre 970-1.440 p. p. m.).

\section{El sitio de Santa Helena en la arqueología regional}

A juzgar por la alta densidad de materiales culturales en el centro de la terraza a lado y lado del antiguo camino a Patillal, en un área de aproximadamente 4 hectáreas, por la presencia de estructuras líticas con fines rituales y la variación de la tipología cerámica, se puede colegir que en la terraza de Santa Helena existieron ocupaciones durante tres momentos. Inicialmente, predominan los tipos cerámicos propios del periodo Loma-Horno, descrito antes, y nuevos elementos tipológicos como naranja liso, café liso, crema a naranja, rojo grano medio. Con menor frecuencia, con respecto a estos, pero de importancia en las tipologías de la tradición Malamboide (Angulo 1981) y Hornoide, se destacan los tipos loma inciso, acanalada y esgrafiada. Esta ocupación se prolonga en el perfil estratigráfico de los cortes 5 y 8, aproximadamente entre 40-120 cm de profundidad, que son los más representativos en materiales y, quizá, en tamaño poblacional. Sobre el incremento y la disminución poblacional para estos periodos, infortunadamente no existen aproximaciones estadísticas precisas (en cuanto a la densidad de tiestos) ni en Colombia (Ardila 1996, 66) ni en Venezuela (Oliver 1989; Sanoja 1981). Pero en la vertiente noroeste de la SNSM hay rastros de una ocupación humana temprana para el mismo momento (principios del I milenio d. C.), cuyas poblaciones emplearon elementos arquitectónicos similares a los de la cultura tairona, hasta la llegada de los conquistadores (Giraldo 2010, 269).

A partir de grandes núcleos y guijarros, la industria lítica se especializó en la elaboración de artefactos de molienda, como manos de moler, bases de molienda de superficie plana, redonda y cóncava, así como otros instrumentos de corte y descame. Es preciso señalar que la tecnología de percusión directa se aprecia en artefactos como lascas triangulares, concoidales y prismáticas, que por su morfofuncionalidad permitieron llevar a cabo la manipulación de estos artefactos en procesos de cortar, raspar y perforar piezas de fauna silvestre. Asimismo, 
la intensidad de ocupación se refleja en los suelos de color oscuro, friables, aptos para la agricultura, asociados a la alta concentración de materiales.

En el segundo momento se observa continuidad con su predecesor; predominan los tipos cerámicos anteriores (naranja liso, café liso, crema a naranja), pero aparece el tipo habano. En El Espinito, más al sur de Santa Helena, se aprecia la conjugación de tradiciones cerámicas antiguas con las formas características de la tradición serrana, en la cual se inscriben las del periodo Portacelli, fechadas en la excavación de El Cercado entre los siglos XII a XV d. C. (tabla 1). Estas fechas son importantes por cuanto nos muestran la relación de los portadores Portacelli con los grupos serranos establecidos en la región (posiblemente taironas). Por otra parte, la industria lítica sigue la tradición desarrollada durante la primera ocupación. Ya cerca de Caracolí, la influencia serrana es más evidente, como se desprende de los hallazgos de ofrendatarios con piedras pulidas (tumas) similares a las del litoral caribe (Dibulla) (figura 5).

Figura 5. Tumas (piedras pulidas) serranas de Caracolí

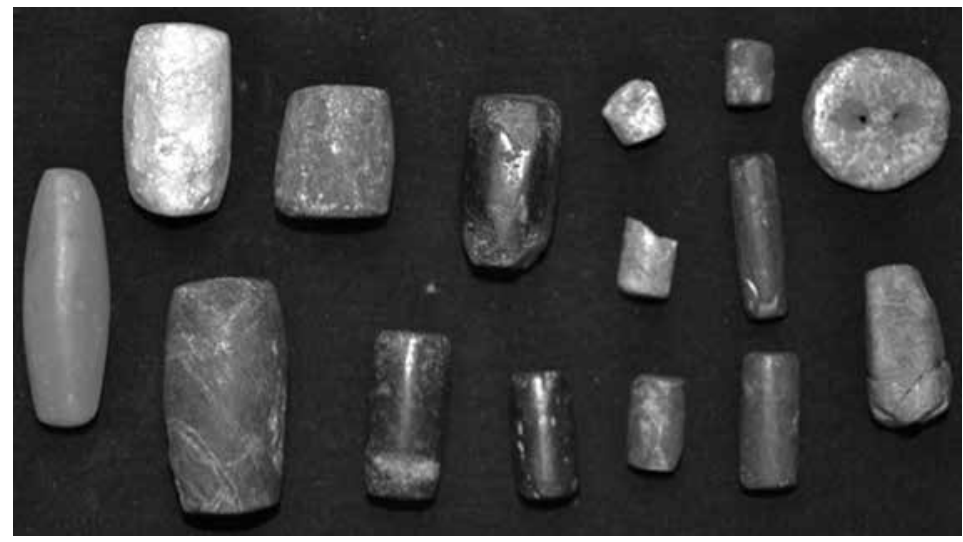

Fuente: elaboración propia.

Hay que anotar que el momento correspondiente a la tradición Ranchoide se concentra en la parte superior de la terraza. Durante este periodo se construyen estructuras líticas con fines rituales y se practican enterramientos primarios directos. Hacia el sur de Santa Helena, en El Espinito 3 (figura 1), las estructuras líticas se emplean en la construcción de plazas para reuniones comunitarias, tal como se acostumbra en la SNSM (Cadavid y Herrera 1985, 5-54; Giraldo 2010, 269; Herrera 1985; Langebaek 2005, 2007; Oyuela 2002, 45-64).

Finalmente, a juzgar por la presencia de cerámica contemporánea, balines de plomo, un crucifijo metálico y huesos de ganado equino, el sitio estuvo 
ocupado hasta mediados del siglo XIX, cuando los indígenas que habitaban este espacio se fueron debido a la presión de agricultores guajiros que llegaron a cultivar tomate y otros productos. Llama la atención la presencia de un antrosol (tierra negra transformada por la gente), que dio origen a un desarrollo agrícola bastante intenso, pues en Colombia este tipo de suelo ha sido reportado primordialmente en la Amazonía (terras pretas do indio TPI). En Santa Helena, el antrosol fue utilizado hasta tiempos históricos, antes de ser abandonado tras la ritualización mediante la construcción de estructuras líticas.

Estos antrosoles se conocen como suelos con clara evidencia de una prolongada ocupación humana, con alta acumulación de basura doméstica (cerámica, carbón, material lítico), en posiciones topográficas con buena visibilidad. Los tenores de fósforo, calcio, magnesio, potasio, $\mathrm{pH}$ y micronutrientes son significativamente mayores que en las áreas adyacentes, y, por ende, la fertilidad es mayor (Morán 1990, 178). En una terraza del río Ranchería en Barrancas se ha reportado el mejoramiento del suelo mediante la aportación de limo del río (Socarrás 2003, 53-97). Al respecto vale la pena resaltar la importancia de la tierra negra (la hija negra de la Madre) mencionada en el mito fundacional de los koguis de la vertiente septentrional de la SNSM, que dio origen a la vida y al poblamiento del mundo de este grupo en un templo especial (Preuss 1993, 10-11). La tierra negra, sin embargo, representa un dilema para los indígenas contemporáneos, pues a pesar de su fertilidad no se usa para sus cultivos, debido a que está prohibido en la tradición serrana, porque, según dicen, hay “mucho diablo por allí” (Reichel-Dolmatoff 1985, I: 105).

A diferencia de otros sitios de la península de La Guajira que contienen una muy baja densidad de artefactos líticos (Ardila 1996, 89-90), El Cercado, El Espinito y Santa Helena (figura 1) sobresalen por la alta concentración de líticos con una amplia variedad de formas y usos, entre los que se destaca su empleo en el procesamiento de alimentos, posiblemente de granos y raíces, a juzgar por la abundancia de los artefactos de molienda (metates, manos). La materia prima para la elaboración de los artefactos líticos proviene tanto del río como de la parte alta de la SNSM. Estos hallazgos desvirtúan la hipótesis sobre la ausencia de manos de moler y metates durante la primera ocupación (Hornoide) y su aparición durante la segunda fase (Ranchoide) (Langebaek, Cuéllar y Dever 1998, 90), lo que sugiere una secuencia yuca-maíz, posición refutada también mediante el análisis de macrorrestos que demuestra la presencia de maíz durante ambas ocupaciones (Socarrás 2003, 94).

También, se destaca el significativo incremento demográfico durante la fase Ranchoide (siglos VI-XVI d. C.) en Santa Helena, a juzgar por la cantidad de 
fragmentos cerámicos, tanto en la prospección como en los cortes estratigráficos (tabla 2), lo que controvierte la idea de un posible abandono hacia el siglo XIII d. C. Al contrario, en la UE 7 de El Cercado se registra una significativa presencia de tipos cerámicos Portacelli.

\section{Conclusiones}

El área inundada por el actual embalse de El Cercado tuvo en tiempos prehispánicos una función múltiple. Por un lado, según la interpretación del mamo wiwa que nos acompañó en la interpretación etnográfica (conocedor de las tradiciones serranas y quien aún hace pagamentos por la Línea Negra de la SNSM), se observa en El Espinito, al lado de rápidos del río Ranchería, evidencias de rituales de ablución y reuniones comunitarias tardías. En El Cercado se aprecia una zona de enterramientos de ofrendas cerámicas (vasijas antropomorfas y con decoración de aves); en Santa Helena se observa una terraza para cultivos intensivos de vegetales desde inicios del I milenio d. C., y también para rituales de pagamento. La influencia serrana se registra en la industria lítica y cerámica y en los rituales de pagamento, en la parte del piedemonte, mientras que la influencia guajira en las tradiciones cerámicas (Hornoide y Ranchoide) es muy significativa. En la cosmovisión de los actuales pueblos de la SNSM, como los koguis, ijkas, wiwas y kankuamos (estos últimos se encuentran en proceso de recuperación de sus tradiciones con mamos wiwas como maestros), la ritualidad mediante ofrendas de pagamento es muy importante. Estas consisten en envoltorios en hojas de maíz de tumas, palo de Brasil, arena de río y otros objetos apreciados, que se colocan debajo de menhires y en otros sitios sagrados alrededor de la Línea Negra, cuya función es el mantenimiento de la vitalidad de la sociedad, su supervivencia, reproducción y protección del territorio ancestral (Uribe 1998, 90). Por lo visto en las prácticas contemporáneas, inferimos que la ritualidad y los pagamentos también fueron trascendentales para los primeros agricultores de la región.

Sin embargo, lo destacable del sitio de Santa Helena es la presencia de un antrosol que fue empleado para labores agrícolas intensivas durante varios centenares de años, entre el I milenio d. C. hasta mediados del siglo XX, cuando fue sepultado y ritualizado con estructuras de piedra para evitar su posterior uso agrícola. De esta manera, la expresión tierras negras debe extenderse a otras regiones del país, entre ellas el piedemonte de la Sierra Nevada de Santa Marta, cuyo desarrollo fue independiente del de la Amazonía. 
Por otro lado, los resultados de las excavaciones realizadas en El Cercado, El Espinito y Santa Helena controvierten la hipótesis de un desmejoramiento generalizado del clima hacia el siglo XIII d. C., que habría ocasionado el abandono de río Ranchería medio. Se observa una reducción de la población en Santa Helena, pero nunca un abandono total de la región.

Los sitios arqueológicos reportados reflejan la confluencia cultural serrana y guajira. La primera en la industria lítica, la segunda en las tradiciones cerámicas (Hornoide y Ranchoide), lo cual sugiere que la población acogió la presencia de materia prima abundante proveniente de la parte alta del río Ranchería, y la influencia cultural de los antiguos pobladores de las sabanas de La Guajira, cuyo medio ambiente fue más propicio para las labores agrícolas prehispánicas, muy diferente al actual paisaje guajiro. De hecho, el sector entre Distracción y Fonseca sigue siendo utilizado en el cultivo de arroz, gracias a la irrigación con las aguas del río Ranchería.

\section{Referencias}

Andrade, Ángela. 1990. "Sistemas agrícolas tradicionales en el Medio Caquetá”. En La selva humanizada. Ecología alternativa en el trópico húmedo colombiano, editado por François Correa, 59-81. Bogotá: ICAN, Fondo FEN, Cerec.

Angulo Valdés, Carlos. 1981. La tradición malambo. Un complejo temprano en el noroeste de Suramérica. Bogotá: Fundación de Investigaciones Arqueológicas Nacionales.

Ardila, Gerardo. 1996. Los tiempos de las conchas. Investigaciones arqueológicas en la costa de la península de La Guajira. Bogotá: Universidad Nacional de Colombia.

Botero, Pedro. 2001. "Relaciones entre las ciencias de la tierra y la arqueología”. En Memorias del Simposio Pueblos y ambientes: una mirada al pasado precolombino, editado por Gaspar Morcote, 7-46. Bogotá: Academia Colombiana de Ciencias Exactas, Físicas y Naturales.

Cadavid, Gilberto y Luisa Fernanda Herrera. 1985. "Manifestaciones culturales en el área tairona”. Informes Antropológicos 1: 5-54.

Castellanos, Juan de. (1601) 1997. Elegías de varones ilustres de Indias. Bogotá: Gerardo Rivas Moreno Editorial.

De Aníbal, Bartolomé et al. (1578) 1983. Relación de los reyes del Valle de Upar. Transcripción de Víctor Manuel Patiño. Cespedesia 45-46: 193-213.

Descola, Philippe. 2012. Más allá de naturaleza y cultura. Buenos Aires: Amorrortu.

Dever, Alejandro. 2007. "Social and Economic Development of a Specialized Community in Chengue, Parque Tairona, Colombia”. Tesis de doctorado en Filosofía, Facultad de Artes y Ciencias, Universidad de Pittsburgh, Pittsburgh. 
Drennan, Robert. 2000. Las sociedades prehispánicas del Alto Magdalena. Bogotá: Instituto Colombiano de Antropología e Historia.

Geraldes, Wenceslau, Gilvan Coimbra Martins, Hednaldo Narciso Lima. 2006. "An Amazon Dark Earth Profile Description from a Site Located in the Floodplain (Várzea) in the Brazilian Amazon”. En Pueblos y paisajes antiguos de la selva amazónica, editado por Gaspar Morcote Ríos, Santiago Mora Camargo y Carlos Franky Calvo, 293-300. Bogotá: Universidad Nacional de Colombia.

Giraldo, Santiago. 2010. "Lords of the Snowy Ranges: Politics Place, and Landscape Transformation in Two Tairona Towns in the Sierra Nevada de Santa Marta, Colombia”. Tesis de Doctorado en Antropología, División de Ciencias Sociales, Universidad de Chicago, Illinois.

Herrera, Luisa Fernanda. 1985. Agricultura y cambios de vegetación en la Sierra Nevada de Santa Marta. Bogotá: FIAN, Banco de la República.

—. 1987. "El manejo del medio ambiente natural por el hombre prehispánico en la Sierra Nevada de Santa Marta”. Boletín del Museo del Oro 19: 83-86.

Hugh-Jones, Stephen. 2012. “Nuestra historia está escrita en las piedras”. En El aliento de la memoria. Antropología e historia en la Amazonía andina, editado por François Correa Rubio, Jean-Pierre Chaumeil y Roberto Pineda Camacho, 29-64. Bogotá: Universidad Nacional de Colombia.

—. 2013. La palma y las pléyades. Bogotá: Universidad Central.

Isaacs, Jorge. (1883) 1951. Estudio sobre las tribus indígenas del Magdalena. Bogotá: Biblioteca Popular de Cultura Colombiana.

Langebaek, Carl. 2005. The Prehispanic Populations of the Santa Marta Bay. Pittsburgh: University of Pittsburgh.

-.2007. Indios y españoles en la Antigua Provincia de Santa Marta. Bogotá: Ediciones Uniandes.

Langebaek, Carl Henrik, Andrea Cuéllar y Alejandro Dever. 1998. Medio ambiente y poblamiento en La Guajira: investigaciones arqueológicas en el Ranchería Medio. Bogotá: Ediciones Uniandes.

Lleras, Roberto. 1987. "La utilización de las áreas libres en Ciudad Perdida”. Boletín del Museo del Oro 19: 97-116.

Mora, Santiago, Luisa Fernanda Herrera, Inés Cavelier y Carlos Rodríguez. 1990. "Suelos antrópicos amazónicos: génesis y dinámica de una organización política”. Revista de Antropología y Arqueología 6 (2): 27-39.

Morán, Emilio. 1990. La ecología humana de los pueblos de la Amazonía. Ciudad de México: Fondo de Cultura Económica.

Morcote, Gaspar. 2008. Antiguos habitantes en ríos de aguas negras. Ecosistemas y cultivos en el interfluvio Amazonas-Putumayo Colombia-Brasil. Bogotá: Universidad Nacional de Colombia.

Morcote, Gaspar y Tomás León. 2012. Las terraspretas del igarapé Takana. Un sistema de cultivo precolombino en Leticia-Amazonas, Colombia. Bogotá: Universidad Nacional de Colombia. 
Morcote, Gaspar, Santiago Mora y Carlos Franky, eds. 2006. Pueblos y paisajes antiguos de la selva amazónica. Bogotá: Universidad Nacional de Colombia.

Oliver, José R. 1989. “The Archaeological, Linguistic and Ethnohistorical Evidence for the Expansion of Arawakan into Northwestern Venezuela and Northeastern Colombia”. Tesis doctoral, Departamento de Antropología. Universidad de Illinois Urbana-Champaign.

Oyuela, Augusto. 2002. "El surgimiento de la rutinización religiosa: la conformación de la élite sacerdotal tairona-kogi”. Arqueología del Área Intermedia 4: 45-64.

Pinheiro, María de Lourdes, Cristine Bastos do Amarante, María de Lourdes Soares, Mariane Furtado, E. L. Nogueira, Regeane da Rocha C. y Brenda Guimaraes. 2006. "Population and Biodiversity in Amazonian Dark Earth Soils”. En Pueblos y paisajes antiguos de la selva amazónica, editado por Gaspar Morcote Ríos, Santiago Mora Camargo y Carlos Franky Calvo, 281-291. Bogotá: Universidad Nacional de Colombia.

Preuss, Konrad Theodor. (1919) 1993. Visita a los indígenas kagaba de la Sierra Nevada de Santa Marta. Observaciones, recopilación de textos y estudios lingüísticos. Bogotá: Instituto Colombiano de Antropología.

Rebellato, Lillian y Heiko Grosch. 2006. "Interpretando la variabilidad cerámica, química y física del suelo en el sitio arqueológico Hatahara, Brasil”. En Pueblos y paisajes antiguos de la selva amazónica, editado por Gaspar Morcote Ríos, Santiago Mora Camargo y Carlos Franky Calvo, 301-310. Bogotá: Universidad Nacional de Colombia.

Reclus, Elisée. 1992. Viaje a la Sierra Nevada de Santa Marta. Bogotá: Biblioteca V Centenario Colcultura, Viajeros por Colombia.

Reichel-Dolmatoff, Gerardo. 1985. Los kogi. Una tribu de la Sierra Nevada de Santa Marta, Colombia. Bogotá: Procultura.

-.1986. Arqueología de Colombia. Un texto introductorio. Bogotá: Lit. Arco, Fundación Segunda Expedición Botánica.

-.1997. Chamanes de la selva pluvial. Ensayos sobre los indios tukano del noroeste amazónico. Devon: Themis Book.

Rodríguez, José Vicente, ed. 2015. El Parque Arqueológico de Facatativá: proceso de recuperación y conservación de la memoria de sus antiguos habitantes. Bogotá: CAR, Universidad Nacional de Colombia.

Rodríguez, José Vicente, Arturo Cifuentes y Francisco Aldana. 2010. Espacios rituales y cotidianos en el alto río Ranchería, La Guajira, Colombia. Arqueología del sureste de la Sierra Nevada de Santa Marta. Bogotá: Universidad Nacional de Colombia, Incoder.

Salas, Rocío. 2011. "La construcción social del paisaje como elemento fundamental de las poblaciones aborígenes del periodo Tardío (500-1550 d. C.) en el Valle del Dorado, Valle del Cauca, Colombia”. Tesis doctoral en Arqueología, Facultad de Ciencias Sociales, Universidad Nacional del Centro de la Provincia de Buenos Aires, Tandil, Olavarría.

Sanoja, Mario. 1981. Los hombres de la yuca y el maíz. Caracas: Monte Ávila.

Socarrás, José Luis. 2003. "Las plantas en la subsistencia de los antiguos habitantes del Medio Ranchería, sur de La Guajira (Colombia)”. Boletín de Arqueología 18: 53-97. 
Uribe, Carlos Alberto. 1998. "De la vitalidad de nuestros hermanos mayores de la Nevada". Revista de Antropología y Arqueología 10 (2): 9-92.

Van der Hammen, María Clara. 1992. El manejo del mundo. Naturaleza y sociedad entre los yukuna de la Amazonía colombiana. Bogotá: Tropenbos.

Vickers, William T. 1989. Los sionas y secoyas. Su adaptación al ambiente amazónico. Quito: Abya Yala.

Vinalesa, José de. 1952. Indios arhuacos de la Sierra Nevada de Santa Marta. Bogotá: Iqueima. 